doi: $10.15407 /$ ujpe62.09.0743

A. ALKIN

Bogolyubov Institute for Theoretical Physics, Nat. Acad. of Sci. of Ukraine

(14b, Metrolohichna Str., Kyiv 03143, Ukraine; e-mail: alkin@bitp.kiev.ua)

\title{
PHENOMENOLOGY OF CHARGED-PARTICLE MULTIPLICITY DISTRIBUTIONS
}

\begin{abstract}
Charged-particle multiplicity distributions are an interesting tool to study both soft- and hardQCD processes in hadronic collisions. Since the last century, a significant range of center-ofmass energies has been probed, ranging from a few GeV to $13 \mathrm{TeV}$ in the latest LHC run. The common analysis of multiplicity distributions at different energies, in different phase space regions, and from sufficiently different experiments provides a way to systematize and to review available phenomenological models of multiple particle production. In this work, a phenomenological model that can describe simultaneously the charged-particle multiplicity distributions in various restricted pseudorapidity intervals for proton-proton collisions is suggested. The model is successfully applied to experimental results of the ALICE experiment at LHC.

Ke ywords: charged-particle multiplicity, proton-proton scattering, LHC.
\end{abstract}

\section{Introduction}

Charged particle multiplicity distributions are basic and general observables in modern collider experiments, by their nature containing information both on soft QCD processes (that still dominate even at LHC energies) and hard scattering, thus allowing one to explore both components and their interrelation. As a direct measure of the collision event inelasticity, the multiplicity potentially contains information on various features of the particle production mechanisms and the hadronization. However, while there is a direct correspondence in heavy ion physics between multiplicity and centrality [1], the situation in proton-proton scattering is much less clear. With the recent unambiguous information on collectivity in pp scattering at LHC energies [2], as well as the potentially non-trivial transverse structure of the interaction region [3], the possible relation between the multiplicity and the underlying interaction becomes even more interesting. However, as usual, when dealing with observables of statistical nature, we must al-

(c) A. ALKIN, 2017

ISSN 2071-0194. Ukr. J. Phys. 2017. Vol. 62, No. 9 ways bear in mind that it can be largely independent of the underlying dynamical process. In this paper, we will explore a straightforward phenomenological model of charged-particle multiplicity distributions in a restricted phase space, which allows disentangling the instrumental limitation of the collider experiments to extract the full phase space charged-particle multiplicity distribution and study its behavior.

\section{Multiplicity Distributions in Collider Experiments}

\subsection{Pre-LHC era}

Both experimental multiplicity measurements and the theoretical attempts to describe them have a rich history. The detailed review of the theoretical understanding of contemporary experimental data was made by Carruthers and Shih in 1987 [4]. Here, we have not repeated the analysis of non-pp multiplicities and old pp data. While the combined analysis of the very wide center-of-mass energy range probed for a few decades of collider experiments may yield interesting results, the limited precision and the multiplicity range of older data would not contribute in 
a significant way. This also means that the available data were not useful in distinguishing and excluding models. This remained mostly true up until a center-of-mass energy of $900 \mathrm{GeV}$ was reached in UA5 experiment and the presence of a possible structure in the multiplicity distribution was identified [5]. It is important to emphasize that this structure was found only in the full phase space multiplicity distribution at the time. A similar observation was later made by the ALICE collaboration at LHC [6] for the same collision energy, but in a restricted phase space distribution. The range of multiplicities in restricted phase space distributions was extended compared to that of UA5, thus indicating that the structure is already present in the central pseudorapidity window at $900 \mathrm{GeV}$. This will be considered in more details in 3.2.

\subsection{Multiplicity in LHC experiments}

Since its start in 2008, LHC produced an enormous amount of data on proton-proton, proton-ion, and ionion collisions at center-of-mass energies ranging from 0.9 to $13 \mathrm{TeV}$ with varying beam conditions. The multiplicity distributions obtained in LHC experiments are thus of special interest, as they are produced with largely unchanged experimental setups and measurement procedures, enabling a combined analysis, in which systematic uncertainties can be controlled in an assumption that they are similar between the measurements at different energies. In an attempt to model the restricted phase space multiplicity distributions, we concentrate on those measured in symmetric $\eta$ regions. From available LHC experiments, only CMS [7] and ALICE [8] have published a large set of data in varying pseudorapidity ranges. ALICE presented measurements at more energies, including $8 \mathrm{TeV}$, for $|\eta|$ up to 1.5 , and presented a preliminary result for the multiplicity distribution measurement in wider ranges, up to $|\eta|<3.4[9]$. Additionally, the effort was made by the ALICE collaboration to refine the non-single-diffractive event sample (i.e., inelastic events excluding single-diffractive events). However, the wide pseudorapidity range data points are not yet available at the time of this publication. ATLAS Collaboration measurements involve a restriction on the particle transverse momentum, whose modeling is beyond the scope of this paper. Measurements at $\sqrt{s}=13 \mathrm{TeV}$ are not yet available as well.

It is important to discuss the event class normalization and the limitations that are inherent in the restricted phase space multiplicity distributions. There is an obvious theoretical preference to dealing with non-single-diffractive event samples (NSD), as diffractive events are known to have properties that are somewhat different from the general inelastic events. However, due to the nature of these events, they have a tendency to have a very low (or no at all) multiplicity in the central (pseudo)rapidity, thus contributing mostly to value of $P(0)(P(N)$ being the restricted phase space multiplicity distribution) and thus only affect the overall normalization. Moreover, excluding the single-diffractive events requires modeling their cross-section fraction and other properties within event generators used to simulate various detector inefficiencies, by increasing, therefore, the systematic uncertainty of a multiplicity distribution. As demonstrated by ALICE [8], the total systematic uncertainties are strongly correlated bin-to-bin and cannot be disentangled in a simple way, by complicating a phenomenological analysis of experimental data. In this paper, we use, however, multiplicity distributions normalized to a nonsingle-diffractive event sample, keeping in mind that any measurement procedure can reliably remove only those diffractive events from the multiplicity distribution, to which the detector set is sensitive. In particular, ALICE only considers events with diffractive system mass $M_{X} \leq 200 \mathrm{GeV}$ [10] as singlediffractive. The residual single-diffractive events in the sample affect the low-multiplicity part of the multiplicity distribution.

As was already mentioned in the previous paragraph, the value $P(0)$ depends mostly on phase space restrictions. This value should be explicitly modeled as the effect of a geometrical restriction on the particle counting region accounting for the spatial distribution of produced particles. However, even without explicit model, this value can be used to put a restriction on the scaling factor introduced by ALICE [8] in a parametrization of the measured distributions. We leave the details to be discussed within the next section.

\section{Models of Multiplicity Distributions}

\subsection{Negative-binomial distribution}

A number of discrete probability distributions were used to model hadronic multiplicities. The most successful description (up to a center-of-mass energy of 
$900 \mathrm{GeV}$ ) was achieved with a single negative binomial discrete probability distribution (NBD) [11]. We will define it here as

$P(n ;\langle n\rangle, k)=\frac{\Gamma(k+n)}{\Gamma(k) \Gamma(n+1)}\left[\frac{\langle n\rangle}{k+\langle n\rangle}\right]^{n}\left[\frac{k}{k+\langle n\rangle}\right]^{k}$,

where $\langle n\rangle$ is the average value, and $k$ is a shape parameter related to the distribution variance $D$ by

$\frac{D^{2}}{\langle n\rangle^{2}}=\frac{1}{\langle n\rangle}+\frac{1}{k}$.

Note that setting $k=1$ yields the Bose-Einstein distribution, while, in the limit $k \rightarrow \infty$, we get the Poisson distribution.

Almost half a century ago, Polyakov [12] and then Koba, Nielsen, and Olesen (KNO) [13] independently suggested that, at a high enough center-of-mass energy, the probability of producing $N$ particles in a collision should reach an asymptotic shape, when expressed as a function of $z=N /\langle N\rangle$,

$P(N)=\frac{1}{\langle N\rangle} \psi(z)$.

While it was initially found to hold for a limited range of multiplicities already at ISR energies [14] for a non-single-diffractive event sample, it was violated for all inelastic events. It was initially found to hold for non-single-diffractive events at energies up to $\sqrt{s}=900 \mathrm{GeV}$ [6]. However, the further analysis [8] showed that it is significantly violated for LHC energies in all available $\eta$ intervals even for NSD events. Using the KNO variable $z$, we can derive the behavior of NBD in the scaling limit defined by

$n \rightarrow \infty, \quad\langle n\rangle \rightarrow \infty, \quad$ fixed $z$,

as $\langle n\rangle / k \gg 1$, formula (1) becomes

$P(n ; k)=\frac{1}{\langle n\rangle} \psi(z ; k)$

with

$\psi(z ; k) \equiv \frac{k^{k}}{\Gamma(k)} z^{k-1} e^{-k z}$

that is a special case of the gamma distribution

$\psi(x ; k, \gamma)=\frac{\gamma^{k}}{\Gamma(k)} x^{k-1} e^{-\gamma x}$, where the shape parameter $\gamma$ coincides with $k$ due to the requirement that $\langle z\rangle=1$. We will use the gamma distribution rewritten with $\langle x\rangle$ as a parameter:

$\psi(x ; k,\langle x\rangle)=\frac{k}{\langle x\rangle} \frac{1}{\Gamma(k)}\left(\frac{k x}{\langle x\rangle}\right)^{k-1} e^{-\frac{k x}{\langle x\rangle}}$.

Based on the properties described above, we will consider a set of possible approximations for the restricted phase space multiplicity distributions, by using combinations of the functions defined above.

\subsection{Structures in multiplicity distributions}

After the inability of a single negative binomial distribution to describe the shape of a multiplicity distribution was discovered, a number of papers examined possible scenarios and their phenomenological consequences, where the multiplicity distribution consists of two incoherent components designated soft and semihard both described by NBDs, with the total distribution being a weighted sum $[15,16]$. These ideas were applied to CMS Collaboration non-singlediffractive multiplicity distributions by Gosh in 2012 [17]. While none of the proposed scenarios was found to be realized, some important conclusions could be derived. The relative importance of the second ("semi-hard") NBD term grows with the center-ofmass energy, as well as the pseudorapidity window size. However, it can be argued that the multiplicity distributions in the most restricted ranges can be suitably well described by a single NBD. The first ("soft") NBD term was found to be almost independent of $\sqrt{s}$ hinting on a possible partial scaling behavior in charged-particle multiplicity distributions. The similar analysis performed by the ALICE Collaboration [8] has confirmed some observations. However, it also emphasized the particular issue with modeling distributions that are strongly correlated bin to bin due to a nature of the measurement. We slightly improve this analysis by introducing a reasonable constraint on the fits and consider other possibilities for both the first and second terms of the weighted sum. Finally, we consider a unified description of the restricted phase space distributions in different $\eta$ ranges for a fixed $\sqrt{s}$ and discuss the possible mechanisms of generating the second term, as well as their phenomenological consequences.

It is worth mentioning that another type of structures in experimental multiplicity distributions was 
identified by examining the recurrent relation representation of the probability distributions $[18,19]$

$(n+1) P(n+1)=\langle n\rangle \sum_{i=0}^{n} C_{i} P(n-i)$.

However, it should be noted that the experimental multiplicity distribution is obtained with regularized unfolding and thus contains residual oscillations with a "period" that is proportional to $\sqrt{n}$, as indicated by the structure of the unfolding response matrix [8]. The observed property of coefficients $C_{i}$ of the recurrent relations to detect an oscillatory structure at low multiplicities is thus can be explained as an artifact of the regularized unfolding [20]. One of the ways to investigate the possibility of physical oscillating structures in multiplicity distributions would be to re-formulate the unfolding problem in terms of recurrent relation coefficients and to study their dependence on statistical fluctuations in the raw data.

\subsection{Modeling multiplicity in a restricted phase space}

\subsubsection{Direct approach}

We will follow the original approach of ALICE [8], that introduces a scaling factor $\lambda$ in a way that

$\lambda \sum_{n=1}^{n_{\max }} P(n ; \mathbf{p})=\sum_{n=1}^{n_{\max }} P_{\exp }(n)$,

i.e. the model probability distribution $P(n ; \mathbf{p})$, with its best-fit parameters $\mathbf{p}$, is re-normalized to be compatible with the experimental distribution in the available range of multiplicities. This is needed to account for the fact that, in the model distributions used, values at $n=0$ are lower than at $n=1,2, \ldots$ up to the distribution peak, while $P_{\exp }(0)$ in the experimental distributions is larger than the rest of the distribution due to phase space restrictions, and, thus, the reminder of the distribution is scaled down. Noting that the overall normalization of the experimental distribution is dependent on the value of $P_{\exp }(0)$, we can add a constraint for the fit criterion based on this value. Using (10) and the fact that both experimental and model distributions are normalized to 1 , we can write

$$
\begin{aligned}
& P_{\exp }(0)+\sum_{n=1}^{n_{\max }} P_{\exp }(n)+\Delta\left(\sum P_{\exp }\right)=1, \\
& \Delta\left(\sum P_{\exp }\right) \approx 0, \\
& P_{\exp }(0)+\lambda \sum_{n=1}^{n_{\max }} P(n ; \mathbf{p}) \approx 1, \\
& \Delta \chi^{2}=\left(\frac{P_{\exp }(0)+\lambda \sum_{n=1}^{n_{\max }} P(n ; \mathbf{p})-1}{\sigma_{0}}\right)^{2},
\end{aligned}
$$

where $\sigma_{0}$ is the uncertainty on $P_{\exp }(0)$, and we neglect the reminder of the distribution sum for $n>$ $>n_{\max }$, as the multiplicity distributions decrease very rapidly. The full fit criterion is written therefore as

$\chi^{2}=\beta \Delta \chi^{2}\left(P_{\exp }(0), P(n ; \mathbf{p})\right)+$

$+\sum_{n=1}^{n_{\max }}\left(\frac{\lambda P(n ; \mathbf{p})-P_{\exp }(n)}{\sigma_{n}}\right)^{2}$,

where we introduce the Lagrange multiplier $\beta$, which will be adjusted to improve fit quality, but will not be considered a free parameter. An obvious function of the factor $\beta$ is to off-set the relatively large $\sigma_{0}$ uncertainty and thus partially compensate for the distribution freedom within the correlation corridor. It was found that the stable convergence is achieved at values $\beta \sim 10^{2}$.

Consider a generic functional form of the multiplicity distribution defined as a weighted sum of two probability distributions $P_{1}, P_{2}$ with their respective

Table 1. Functional forms of the weighted sum components as per (16) with the typical $\chi^{2}$ values.

Note that, due to the significant correlated uncertainties in experimental distributions, these values are at least one order of magnitude lower than one would normally expect

\begin{tabular}{|c|l|l|c|}
\hline No. & $P_{1}$ & $P_{2}$ & typical $\chi^{2} / \mathrm{NDF}$ \\
\hline 1 & NBD & NBD & 0.1 \\
2 & Poisson & NBD & 10 \\
3 & NBD & Bose-Einstein & 10 \\
4 & NBD & Gamma & 0.1 \\
\hline
\end{tabular}

ISSN 2071-0194. Ukr. J. Phys. 2017. Vol. 62, No. 9 
Table 2. Best-fit parameter values for the NBD + NBD and NBD + Gamma

model distributions for all $\sqrt{s}$ and $\eta$ ranges considered. Note that the uncertainties of parameters are calculated, by assuming uncorrelated errors in the experimental distribution, and thus are unreliable

\begin{tabular}{|c|c|c|c|c|c|c|c|c|}
\hline \multicolumn{9}{|c|}{$\mathrm{NBD}+\mathrm{NBD}$} \\
\hline$\sqrt{s}(\mathrm{TeV})$ & $|\eta|<$ & $\lambda$ & $\alpha$ & $\langle n\rangle_{1}$ & $k_{1}$ & $\langle n\rangle_{2}$ & $k_{2}$ & $\chi^{2} / \mathrm{NDF}$ \\
\hline \multirow[t]{3}{*}{0.9} & 0.5 & $0.93 \pm 0.06$ & $0.43 \pm 0.53$ & $2.1 \pm 1.9$ & $3.3 \pm 13.9$ & $5 \pm 4$ & $2.8 \pm 3.4$ & $0.167 / 30$ \\
\hline & 1 & $0.94 \pm 0.02$ & $0.58 \pm 0.25$ & $5.0 \pm 2.4$ & $2.9 \pm 1.7$ & $13 \pm 6$ & $3.8 \pm 3.0$ & $0.162 / 54$ \\
\hline & 1.5 & $0.96 \pm 0.01$ & $0.76 \pm 0.28$ & $9.0 \pm 5.1$ & $2.4 \pm 1.1$ & $22 \pm 14$ & $5.2 \pm 5.6$ & $3.415 / 66$ \\
\hline \multirow[t]{3}{*}{2.76} & 0.5 & $0.93 \pm 0.03$ & $0.50 \pm 0.16$ & $2.5 \pm 1.0$ & $2.8 \pm 2.4$ & $7 \pm 2$ & $3.0 \pm 1.2$ & $0.421 / 44$ \\
\hline & 1 & $0.94 \pm 0.01$ & $0.55 \pm 0.09$ & $5.3 \pm 1.2$ & $2.7 \pm 0.9$ & $16 \pm 2$ & $3.5 \pm 0.9$ & $0.403 / 77$ \\
\hline & 1.5 & $0.95 \pm 0.01$ & $0.68 \pm 0.15$ & $9.8 \pm 3.6$ & $2.2 \pm 0.6$ & $27 \pm 8$ & $4.4 \pm 2.4$ & $6.598 / 99$ \\
\hline \multirow[t]{3}{*}{7} & 0.5 & $0.94 \pm 0.02$ & $0.70 \pm 0.12$ & $3.6 \pm 1.4$ & $1.8 \pm 0.7$ & $12 \pm 3$ & $4.1 \pm 1.6$ & $0.874 / 62$ \\
\hline & 1 & $0.94 \pm 0.01$ & $0.66 \pm 0.05$ & $7.0 \pm 1.1$ & $2.0 \pm 0.4$ & $23 \pm 2$ & $4.2 \pm 0.7$ & $1.871 / 110$ \\
\hline & 1.5 & $0.95 \pm 0.01$ & $0.60 \pm 0.04$ & $9.9 \pm 1.2$ & $2.1 \pm 0.3$ & $32 \pm 3$ & $3.7 \pm 0.5$ & \\
\hline \multirow[t]{3}{*}{8} & 0.5 & $0.93 \pm 0.02$ & $0.57 \pm 0.10$ & $3.1 \pm 1.0$ & $2.0 \pm 1.0$ & $11 \pm 2$ & $3.2 \pm 1.0$ & $0.837 / 60$ \\
\hline & 1 & $0.93 \pm 0.01$ & $0.61 \pm 0.05$ & $6.6 \pm 1.2$ & $2.1 \pm 0.4$ & $22 \pm 2$ & $3.8 \pm 0.7$ & $1.491 / 106$ \\
\hline & 1.5 & $0.94 \pm 0.01$ & $0.70 \pm 0.06$ & $11.6 \pm 2.2$ & $1.8 \pm 0.2$ & $37 \pm 5$ & $4.6 \pm 1.1$ & $5.976 / 138$ \\
\hline
\end{tabular}

\begin{tabular}{|c|c|c|c|c|c|c|c|c|}
\hline \multicolumn{9}{|c|}{$\mathrm{NBD}+$ Gamma } \\
\hline$\sqrt{s}(\mathrm{TeV})$ & $|\eta|<$ & $\lambda$ & $\alpha$ & $\langle n\rangle_{1}$ & $k_{1}$ & $k_{2}$ & $\langle n\rangle_{2}$ & $\chi^{2} / \mathrm{NDF}$ \\
\hline \multirow[t]{3}{*}{0.9} & 0.5 & $0.62 \pm 0.05$ & $0.80 \pm 0.02$ & $5.6 \pm 0.4$ & $2.96 \pm 0.45$ & $2 \pm 1$ & $2.6 \pm 0.4$ & $0.155 / 30$ \\
\hline & 1 & $0.84 \pm 0.20$ & $0.70 \pm 0.20$ & $5.0 \pm 2.8$ & $2.91 \pm 1.53$ & $4 \pm 3$ & $13.7 \pm 6.8$ & $0.162 / 54$ \\
\hline & 1.5 & $0.88 \pm 0.19$ & $0.89 \pm 0.09$ & $9.5 \pm 4.1$ & $2.34 \pm 0.95$ & $5 \pm 4$ & $24.7 \pm 12.0$ & $3.404 / 66$ \\
\hline \multirow[t]{3}{*}{2.76} & 0.5 & $0.98 \pm 0.06$ & $0.55 \pm 0.15$ & $2.5 \pm 1.1$ & $2.66 \pm 1.84$ & $3 \pm 1$ & $8.3 \pm 2.2$ & $0.414 / 44$ \\
\hline & 1 & $0.79 \pm 0.07$ & $0.70 \pm 0.07$ & $5.4 \pm 1.3$ & $2.65 \pm 0.80$ & $3 \pm 1$ & $16.7 \pm 2.7$ & $0.407 / 77$ \\
\hline & 1.5 & $0.80 \pm 0.22$ & $0.86 \pm 0.10$ & $10.1 \pm 4.9$ & $2.18 \pm 0.76$ & $4 \pm 3$ & $28.1 \pm 11.5$ & $6.594 / 99$ \\
\hline \multirow[t]{3}{*}{7} & 0.5 & $0.94 \pm 0.02$ & $0.76 \pm 0.12$ & $3.9 \pm 1.7$ & $1.74 \pm 0.74$ & $4 \pm 2$ & $12.9 \pm 3.8$ & $0.877 / 62$ \\
\hline & 1 & $0.82 \pm 0.04$ & $0.81 \pm 0.04$ & $7.3 \pm 1.2$ & $1.95 \pm 0.36$ & $4 \pm 1$ & $24.0 \pm 2.5$ & $1.915 / 110$ \\
\hline & 1.5 & $0.72 \pm 0.05$ & $0.82 \pm 0.03$ & $10.0 \pm 1.3$ & $2.10 \pm 0.30$ & $4 \pm 1$ & $32.5 \pm 2.8$ & $8.012 / 146$ \\
\hline \multirow[t]{3}{*}{8} & 0.5 & $0.92 \pm 0.03$ & $0.64 \pm 0.11$ & $3.2 \pm 1.1$ & $1.98 \pm 0.89$ & $3 \pm 1$ & $11.5 \pm 2.3$ & $0.830 / 60$ \\
\hline & 1 & $0.77 \pm 0.05$ & $0.77 \pm 0.04$ & $6.8 \pm 1.3$ & $2.12 \pm 0.42$ & $4 \pm 1$ & $23.4 \pm 2.6$ & $1.538 / 106$ \\
\hline & 1.5 & $0.77 \pm 0.07$ & $0.87 \pm 0.03$ & $11.9 \pm 2.2$ & $1.80 \pm 0.24$ & $4 \pm 1$ & $37.9 \pm 4.9$ & $5.974 / 138$ \\
\hline
\end{tabular}

parameter sets $\mathbf{p}_{1}, \mathbf{p}_{2}$

$$
\begin{aligned}
& P(n ; \mathbf{p})=\alpha P_{1}\left(n ; \mathbf{p}_{1}\right)+(1-\alpha) P_{2}\left(n ; \mathbf{p}_{2}\right), \\
& 0<\alpha<1 .
\end{aligned}
$$

The combinations of the probability distributions, used in this analysis, are presented in Table 1 together with their typical $\chi^{2}$ values. Note that the Bose-Einstein distribution can be only used as the ISSN 2071-0194. Ukr. J. Phys. 2017. Vol. 62, No. 9 second term, as it lacks a distinct peak that is present in the experimental multiplicity distribution at low $n$, while the Poisson distribution is too narrow to be used as the second term. The gamma distribution is used in its two-parameter form (8). Only the sum of two NBDs and the sum of NBD and the gamma distribution are in agreement with data, providing a similar description. The best-fit parameters for these two cases are presented in Table 2 for all $\sqrt{s}$ and $\eta$ 
ranges considered. A trend can be identified in $\chi^{2}$ values, namely, the apparent reduction in the fit quality with extending the $\eta$ window. This can be attributed to the fact that the fraction of residual single- and normal double-diffractive events, which have non-zero multiplicity, increases in the given pseudorapidity region with its size changing the multiplicity distribution behavior at low $n$, where the contribution to $\chi^{2}$ is the largest. It is important to emphasize here that, while the parameter values themselves do provide a reasonable description of experimental curves, the uncertainties should be considered with great caution due to correlations within systematic uncertainties of experimental curves. While the best-fit parameters for the sum of two NBDs differ slightly from those reported by ALICE [8], the curves they define lie within the correlation corridors provided with the experimental curves.

As expected, no clear trend is observed in the parameter evolution, both as a function of the energy and pseudorapidity window. This is consistent with the earlier analysis [17] and confirms that the overall multiplicity distribution cannot be unambiguously separated into different event classes with different multiple particle production mechanisms based on the distribution shape alone. This is due to a significant overlap in multiplicity ranges between the two components, which increases the range of weight $\alpha$ variation. However, a simultaneous description of multiplicity distributions in several pseudorapidity ranges is expected to be more reliable for this purpose.

The quality of description with a weighted sum of NBD and the gamma distribution is on par with that of two NBDs. We note that, within uncertainties, the parameter $k \approx 4$ of the gamma term seems to be independent of the center-of-mass energy, which is consistent with a scaling limit for the second component reached already at $\sqrt{s}=0.9 \mathrm{TeV}$. More precise measurements of multiplicity distributions are required to test this observation.

\subsubsection{Unified modeling}

a. Multiplicity reduction model. As was already mentioned in the discussion above, the values of reduced multiplicities in a restricted phase space are determined by the interplay between the restriction itself and the underlying spatial distribution of particles produced in a collision. Let us consider this effect in more details. Let us assume that there is an unrestricted multiplicity distribution $P_{\text {tot }}(N)$, where $N$ is the total number of charged particles produced in an inelastic collision. Let $P(n \mid N, \Delta \eta)$ denote the conditional probability that $n$ charged particles of all $N$ ones fit into a given $\Delta \eta$. We can calculate the average number of charged particles in the $\Delta \eta$ region, when $N$ is fixed, as

$\langle n\rangle_{\Delta \eta, N}=\sum_{n \leq N} n P(n \mid N, \Delta \eta)$

and the overall average as

$\langle n\rangle_{\Delta \eta}=\sum_{N}\langle n\rangle_{\Delta \eta, N} P_{\mathrm{tot}}(N)=$
$=\sum_{N} \sum_{n \leq N} n P(n \mid N, \Delta \eta) P_{\mathrm{tot}}(N)$.

Spanning $P(n \mid N, \Delta \eta)$ in such a way that $P(n>N \mid N, \Delta \eta) \equiv 0$, we can change the order of summation in (18) so it becomes

$\langle n\rangle_{\Delta \eta}=\sum_{n} n\left(\sum_{N} P(n \mid N, \Delta \eta) P_{\mathrm{tot}}(N)\right)$,

and, thus, the quantity

$P_{\text {mod }}(n, \Delta \eta) \equiv \sum_{N} P(n \mid N, \Delta \eta) P_{\text {tot }}(N)$

is the model for the restricted phase space multiplicity distribution we are interested in.

It is important to emphasize here that, to get the structure similar to one in the observed distribution $[8,9]$ with the use of $(20)$, one of the compound distribution components, either total or conditional probability distribution, has to contain a similar structure. As the characteristic feature of multiplicity distribution is present for the full phase space and becomes less apparent with decreasing the pseudorapidity window, we will assume that $P_{\text {tot }}(N)$ is its source. It should be noted that the hypothetical production mechanisms that correspond to the event subsamples identified through a multiplicity distribution differ most likely both in the amount of particles produced in a typical act and the spatial configuration of the produced system. Thus, the factorization assumed in (18) does not hold. As an initial approximation, we will, however, continue operating under 
the assumption that the hypothetical additional particle production mechanism generates the same spatial distribution on average.

In order that (20) be useful, we must make some assumptions about the form of $P(n \mid N, \Delta \eta)$. We need to define its shape with the requirement that $n \leq N$ and that the average observed multiplicity is a function of $N,\langle n\rangle=F(N, \Delta \eta)$. In a borderline case of unrestricted $\eta$ range, this distribution should collapse into an infinitely thin peak at $\langle n\rangle=N$. Assuming the shape of $1 / n_{\text {events }} \mathrm{d} n_{\text {particles }} /\left.\mathrm{d} \eta\right|_{N}$ does not depend on $N,{ }^{1}$ we can start with a linear relation

$\langle n\rangle=A(\Delta \eta) N$,

where the constant parameter $A$ has simple meaning of the area fraction under the pseudorapidity density curve within the $\Delta \eta$ interval, by depending, in a chosen approximation, only on the size and position of this interval. A straightforward choice of $P(n \mid N)$ shape is thus a binomial distribution

$P(n \mid N)=\frac{N !}{n !(N-n) !} p^{n}(1-p)^{(N-n)}$

with mean $\langle n\rangle=N p$ and variance $D=N p(1-p)$ that identifies $p \equiv A$.

b. Full phase space multiplicity distribution. With the explicit form of $P(n \mid N, \Delta \eta)$ in place, we can fit one model of $P_{\text {tot }}(N)$ to a set of measurements in different pseudorapidity intervals at a given center-of-mass energy $\sqrt{s}$.

The choice of a binomial restriction probability distribution (22), in particular, is convenient, if the full distribution $P_{\text {tot }}(N)$ is a NBD or a sum of NBDs. Indeed, substituting (1) into (20), we get

$$
\begin{aligned}
& P(n)=\sum_{N \geq n}\left(\begin{array}{l}
N \\
n
\end{array}\right) p^{n}(1-p)^{N-n} \times \\
& \times\left(\begin{array}{c}
N+k-1 \\
N
\end{array}\right) q^{N}(1-q)^{k},
\end{aligned}
$$

where we let

$q \equiv \frac{\langle N\rangle}{k+\langle N\rangle}$.

\footnotetext{
1 This can be experimentally verified in the future; however, there is no multiplicity-binned measurements of the pseudorapidity density available for the proton-proton scattering at the time of the publication.
}

Table 3. Ratios of average multiplicities in different $\boldsymbol{\eta}$ ranges for distributions normalized to non-single-diffractive events as measured by ALICE [8]

\begin{tabular}{|l|c|c|}
\hline $\begin{array}{c}\sqrt{s} \\
(\mathrm{TeV})\end{array}$ & $\begin{array}{c}\langle n\rangle_{|\eta|<} \\
0.5: 1: 1.5\end{array}$ & Ratio $^{a}$ \\
\hline 0.9 & $3.8: 7.8: 11.8$ & $1: 2.05: 3.11$ \\
2.76 & $4.6: 9.4: 14.2$ & $1: 2.04: 3.09$ \\
7 & $5.7: 11.6: 17.5$ & $1: 2.04: 3.07$ \\
8 & $5.8: 11.9: 17.8$ & $1: 2.05: 3.07$ \\
\hline
\end{tabular}

${ }^{a}$ It should be noted that these ratios are very similar to just the ratios of $\Delta \eta$ ranges indicating that the pseudorapidity density is rather flat in the central region.

This series, in fact, converges to NBD

$P(n)=\left(\begin{array}{c}n+k-1 \\ n\end{array}\right) z^{n}(1-z)^{k}$

with the same parameter $k$ and

$z \equiv \frac{q p}{1-q+q p}$

from which we recover $\langle n\rangle=p\langle N\rangle$. An obvious conclusion is that the apparent double-NBD behavior of restricted phase space multiplicity distributions in this framework follows directly from the similar structure of the unrestricted distribution. It should be noted here that the above derivation is done for an integer $k$. However, it can be shown to hold for real-valued $k$ as well.

The considerations described above allow us to draw some immediate conclusions. From the ratios of average multiplicities in ALICE measurements [8] (see Table 3 ), we can estimate the expected ratios ${ }^{2}$ $A_{1}: A_{2}: A_{3}$. The reduction procedure leaves the parameter (or parameters, in case of a weighted sum) $k$ of the original NBD intact. The shape parameter $k$ within the clan model [11] is related to the average number of particle clans

$N_{\text {clans }}=k \times \ln \left(1+\frac{\langle n\rangle}{k}\right)$,

with the average number of particles per clan

$n_{\text {particles }}=\frac{\langle n\rangle}{N_{\text {clans }}}$

${ }^{2} A_{1,2,3}$ is a shorthand for $A_{-0.5}^{+0.5}, A_{-1}^{+1}$, and $A_{-1.5}^{+1.5}$. 
and, thus, is expected to be determined solely by the underlying production mechanism and not by the pseudorapidity window size. On the other hand, $k$ can be related directly to the two-particle correlation function [11]. Ability (or inability) to describe all restricted phase space multiplicity distributions with one set of shape parameters (for a given weighted sum unrestricted multiplicity distribution model) is thus an important clue into the average spatial configuration of a particle family produced in a hadronic collision.

Additionally, we will not limit the total number of charged particles to even numbers, as is required from the electric charge conservation, as this particular model is only aimed to describe the bulk behavior of the multiplicity distribution. In particular, it does not describe the values at restricted $n=0$ and in first few bins. This choice is related to the leading particle effect in the sense that we describe the particle production excluding the leading particle (or particles), and, thus, the "total" charged-particle multiplicity $N$ can take odd values.

\section{c. Application to ALICE measurements.} From the known behavior of NBD, we can conclude that the instrumental bin $n=0$ cannot be described by this procedure. Let us consider the weighted sum model for $P_{\text {tot }}(N)$ (16) with NBDs. Since the parameter $k$ is unaffected by the binomial reduction, the distinct maximum from the first term will be present in $P_{\text {mod }}$, and, thus, the value of $P_{\bmod }(0)$ will be lower than for the $n>0$ around the maximum.

However, the ALICE results [8,9] indicate an "S" shape at the start of the distribution with the value of $P_{\exp }(0)$ larger than the rest of the distribution even for a non-single-diffractive event sample, especially in large pseudorapidity intervals and at higher $\sqrt{s}$. Such behavior already seen in increasing $\chi^{2}$ for the direct fits of distributions in the central $\eta$ region can be argued to emerge from the double-diffractive and residual single-diffractive events present in the sample. Modeling the effects from such events goes beyond the scope of this paper. However, we note that it can be done within the proposed framework by introducing a separate term into $P_{\text {tot }}(N)$ with a specific $P(n \mid N, \Delta \eta)$ that is based on a different pseudorapidity density. A viable option is the $3^{\text {rd }}$ term in the weighted sum (16) with low $\langle N\rangle$ and the parameter $k \gtrsim 1$ (thus lacking the structure with a max- imum) with a very low reduction factor $A$, so that most of its effect is concentrated at a low multiplicity $n$. Using the measurements from different experimental collaborations which have detector sets with different diffractive mass sensitivity (such as ALICE and CMS) can provide important constraints for that purpose. This will be considered in the follow-up analysis.

Here, we concentrate on modeling the bulk distribution, thus excluding bin $n=0$ from the fit criterion. It was found that the scaling factor similar to one introduced for a direct modeling is not needed in this formulation ${ }^{3}$, indirectly confirming that we are modeling only a subset of events included in the ALICE NSD sample. The fit criterion for a given energy $\sqrt{s}$ is written as follows:

$\chi^{2}=N_{\text {total }} \sum_{\Delta \eta} \frac{1}{N_{\text {bins }}(\Delta \eta)} \chi_{\Delta \eta}^{2}$,

where, for each pseudorapidity range $\Delta \eta$,

$\chi_{\Delta \eta}^{2}=\sum_{n=1}^{n_{\max }}\left(\frac{P_{\bmod }(n, \Delta \eta)-P_{\exp }(n, \Delta \eta)}{\sigma_{n}}\right)^{2}$

and the factor

$N_{\text {total }}=\sum_{\Delta \eta} N_{\text {bins }}(\Delta \eta)$

is intended to restore the meaning of $\chi^{2}$ as a sum of $N_{\text {total }}$ squared deviations from normally distributed random variables. The weighting of criteria for separate $\eta$ ranges is intended to balance the constraints. No weighting the experimental distribution at the largest $\Delta \eta$ will have a more contribution to the total $\chi^{2}$ due to the largest number of experimental points. However, it is also less precise due to a smaller event sample. Unfortunately, due to highly correlated uncertainties in the experimental distribution, the simple criterion is insufficient to estimate the uncertainties on fit parameters reliably, and, without data in more $\eta$ intervals, the fit is rather underconstrained.

It should be noted that the fit criterion (29) does not account for a direct correlation between the parameters $A_{1,2,3}$ and the unrestricted phase space average multiplicity given by the parameters $\langle N\rangle_{1,2}$. In

3 This is mostly due to the fact that, for a non-singlediffractive sample, its value is $\approx 0.94$ that is very close to 1 . 
fact, any (small enough) multiplicative change simultaneously in all the $A_{1,2,3}$ parameters would immediately translate into the reversed change in $\langle N\rangle_{1,2}$ without affecting the fit criterion. This additional freedom in the parameter choice requires limiting the average full phase space multiplicity by some external means. Let us firstly consider the extreme cases. A decrease in the total average multiplicity yields a fit compatible with data until the parameter $A$ for the largest pseudorapidity interval reaches the limiting value of 1 , and, thus, the further decrease in the average unrestricted multiplicity will make the description of the largest $\eta$ range inadequate. An increase in the average multiplicity is not limited by this particular method. However, it is rather obvious that there are physical limitations on how many particles can be produced in a hadron collision at a certain energy. It is possible to estimate the upper limit, though it is quite large for the $\mathrm{TeV}$-scale collider.

This problem can be solved by placing reasonable restrictions on the parameters $A_{1,2,3}$. By definition, $A_{1,2,3}$ are determined by the (assumed) universal pseudorapidity density functional form asymptotic behavior. In this publication, we estimate these parameters through the parametrized $\mathrm{d} N / \mathrm{d} \eta$ distributions (see Fig. 1) fitted to ALICE data from the same dataset [8]. The initial values of parameters are presented in Table 4.

We start with the simplest functional form for the rapidity density:

$\frac{\mathrm{d} N}{\mathrm{~d} y}=\left.\langle N\rangle\right|_{\eta=0}\left(1-\frac{y}{y_{\max }}\right)^{2 k}\left(1+\frac{y}{y_{\max }}\right)^{2 k}$,

where $y_{\max }=\ln \frac{\sqrt{s}}{m_{\mathrm{p}}}$ ( $m_{\mathrm{p}}$ is a proton mass), $k=1$, and $\left.\langle N\rangle\right|_{\eta=0}$ is the particle density at $\eta=0$. Note that this particular type of the function unambiguously arises from the triple pole pomeron model [21,22]. For a given particle with mass $m$ and transverse momentum $p_{\perp}$, we can relate the rapidity density to the pseudorapidity density as

$\frac{\mathrm{d} N}{\mathrm{~d} \eta}=\frac{1}{\sqrt{1+\frac{1}{b^{2} \cosh ^{2} \eta}}} \frac{\mathrm{d} N}{\mathrm{~d} y}$

with $b=p_{\perp} / m$ and

$y=\ln \left(\frac{\sqrt{1+b^{2} \cosh ^{2} \eta}+b \sinh \eta}{\sqrt{1+b^{2}}}\right)$.

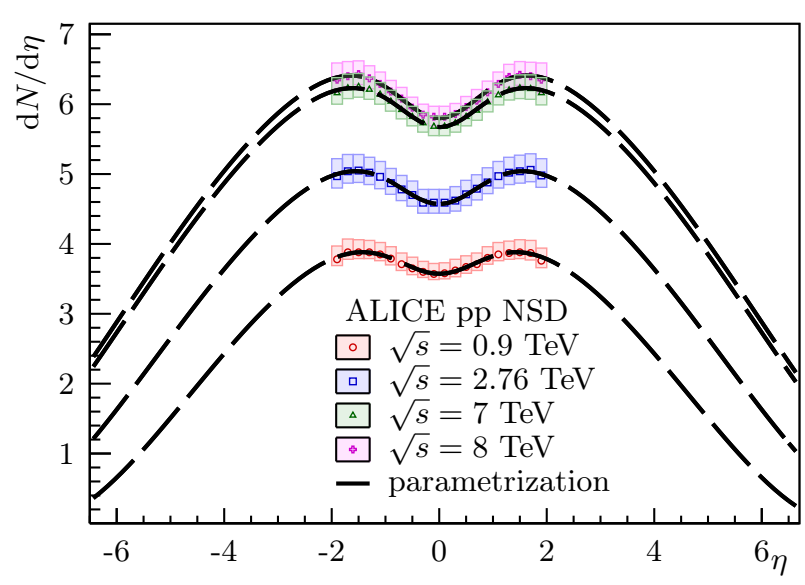

Fig. 1. Parametrization of the $\mathrm{d} N / \mathrm{d} \eta$ distributions from ALICE with Eqs. (32)-(34) to extract initial values for $A_{1,2,3}$

Using an "effective" $b$ as a free parameter, we can easily fit the pseudorapidity density data at all energies with $b \approx 0.6$. The extrapolation should be considered with caution, as it completely neglects the non-perturbative (in particular, diffractive) contributions. The apparent low value of $b$ is also of interest, but it emerges as a non-trivial convolution of both $p_{\perp}$ and the mass spectrum of produced particles and, thus, does not have a straightforward interpretation.

Note that the estimated ratios are quite close to those observed in average multiplicities, but the estimated $A_{3} / A_{1}$ increases, while $\langle n\rangle_{3} /\langle n\rangle_{1}$ decreases, as the energy increases, by indicating the widening of a pseudorapidity density shape that is not seen in the simple parametrization. This analysis will be redone as soon as new measurements (in particular, multiplicity distributions in additional pseudorapidity intervals) will become available.

Due to the significant correlated errors of the experimental distribution, parameters other than the average multiplicity of the full phase space multiplic-

Table 4. Estimated values for $\boldsymbol{A}_{\mathbf{1}, \mathbf{2}, \mathbf{3}}$

from the extrapolated parametrization of ALICE NSD $\mathrm{d} N / \mathrm{d} \eta$ measurements [8]

\begin{tabular}{|c|c|c|}
\hline$\sqrt{s}(\mathrm{TeV})$ & $A_{-0.5}^{+0.5}: A_{-1}^{+1}: A_{-1.5}^{+1.5}$ & Ratio \\
\hline 0.9 & $0.104: 0.212: 0.323$ & $1: 2.040: 3.112$ \\
2.76 & $0.091: 0.186: 0.284$ & $1: 2.044: 3.127$ \\
7 & $0.083: 0.170: 0.260$ & $1: 2.041: 3.119$ \\
8 & $0.082: 0.168: 0.257$ & $1: 2.043: 3.126$ \\
\hline
\end{tabular}



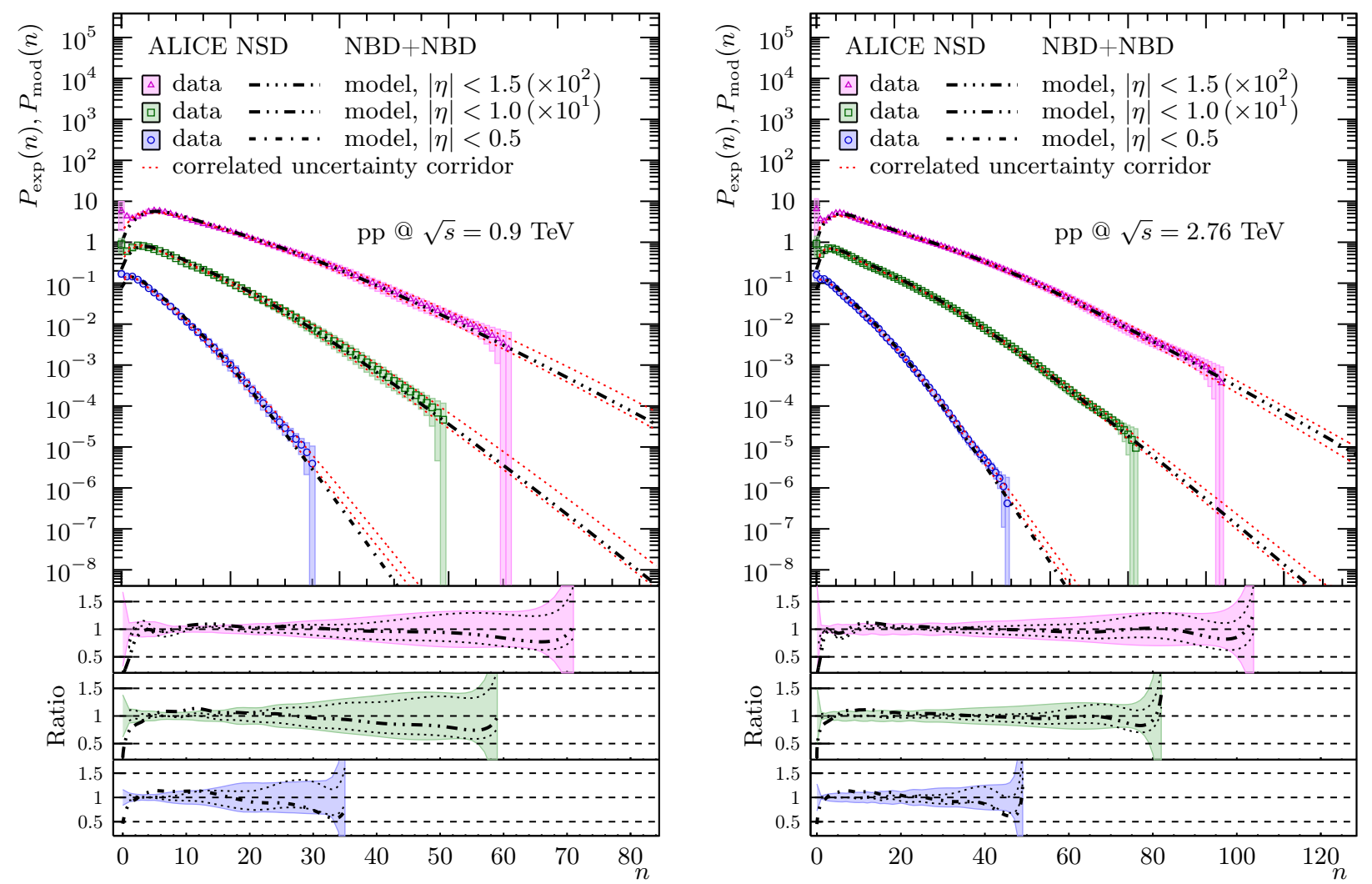

Fig. 2. Model fit of the ALICE non-single-diffractive multiplicity distributions in proton-proton collisions within the weighted NBD sum model for the full phase space distribution and the binomial reduction at $\sqrt{s}=0.9$ (left) and 2.76 (right) TeV. Bounding direct double-NBD fits from ALICE are added to indicate a correlated uncertainty corridor. Curves at different $\eta$ intervals are displaced vertically for clarity. Shaded areas indicate the combined statistical and systematic uncertainties of experimental data. Ratios of model fits to experimental data are presented in the bottom of the figure with the same convention for the shaded area

ity distribution are largely unrestricted, and the usual fit procedure is unable to produce a reliable uncertainty estimate on fit parameters. Thus, we will avoid drawing any rigorous conclusions in this publication based on the parameter evolution. We will outline, however, the direction of theoretical and experimental inquiries that can be pursued to verify and to improve the methods described here.

The resulting model fit with $P_{\text {tot }}(N)$ defined by a weighted sum of two NBDs is presented in Figs. 2 and 3 , and the corresponding parameters are given in Table 5. The weighted sum of NBD and the gamma distribution also provides the simultaneous description of all three restricted phase space multiplicities (see Table 5), however, with less compatibility, especially at low center-of-mass energies. It also features a somewhat steeper growth of the unrestricted phase space average multiplicity with $\sqrt{s}$ (see Fig. 4). But, within the expected uncertainty, we may say that these trends are similar. We note that, due to a low fit quality for the NBD + Gamma option, there is a considerably more freedom in determining the $\langle N\rangle_{1,2}$ and $\alpha$ parameters, and the apparent trend may be changed. However, the result for NBD $+\mathrm{NBD}$ is more reliable and stable. The overall unrestricted multiplicity distributions for energies from 0.9 to $8 \mathrm{TeV}$ extrapolated from the NBD + NBD and NBD + Gamma models are presented in Fig. 5.

\subsection{Discussion of results}

First of all, let us reiterate once again the fact that, due to experimental limitations, specifically to the correlated uncertainties, we are unable to extract errors on model parameters without more data and additional restrictions from different observables. In particular, we cannot draw rigorous conclusions from 

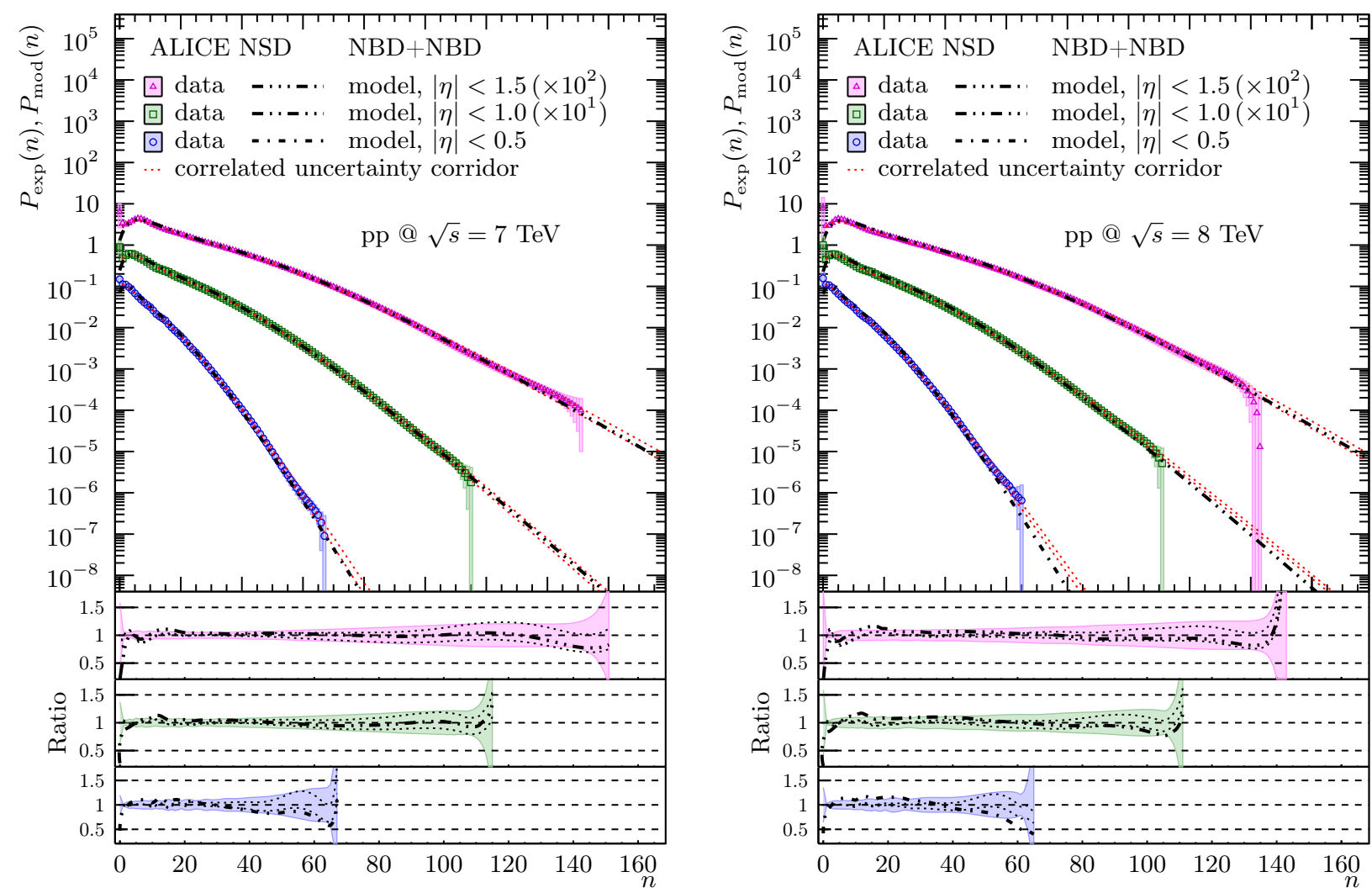

Fig. 3. Model fit of the ALICE non-single-diffractive multiplicity distributions in proton-proton collisions within the weighted NBD sum model for the full phase space distribution and the binomial reduction at $\sqrt{s}=7$ (left) and 8 (right) TeV. Bounding direct double-NBD fits from ALICE are added to indicate a correlated uncertainty corridor. Curves at different $\eta$ intervals are displaced vertically for clarity. Shaded areas indicate the combined statistical and systematic uncertainties of experimental data. Ratios of model fits to experimental data are presented in the bottom of the figure with the same convention for the shaded area

the trends of shape parameters $k$ for both the NB and gamma distributions, as those may not hold after repeating this analysis with more data. However, we will discuss the possible physical interpretations of the observed behavior.

The restriction factors $A_{1,2,3}$ differ between the double-NBD and NBD + Gamma models. In both cases, they decrease with energy, as well as their ratios. However, the ratios differ both from those estimated by the pseudorapidity density parametrization and the ratios of average multiplicities. Note that, due to the fact that the low-multiplicity part of the distribution is not fully modeled, the projected average multiplicity is slightly higher, which can explain the apparent difference in ratios. It can confirm also the observation from the parametrized pseudorapidity density that its shape widens with the growth of $\sqrt{s}$ and may be wider than our parametriza-

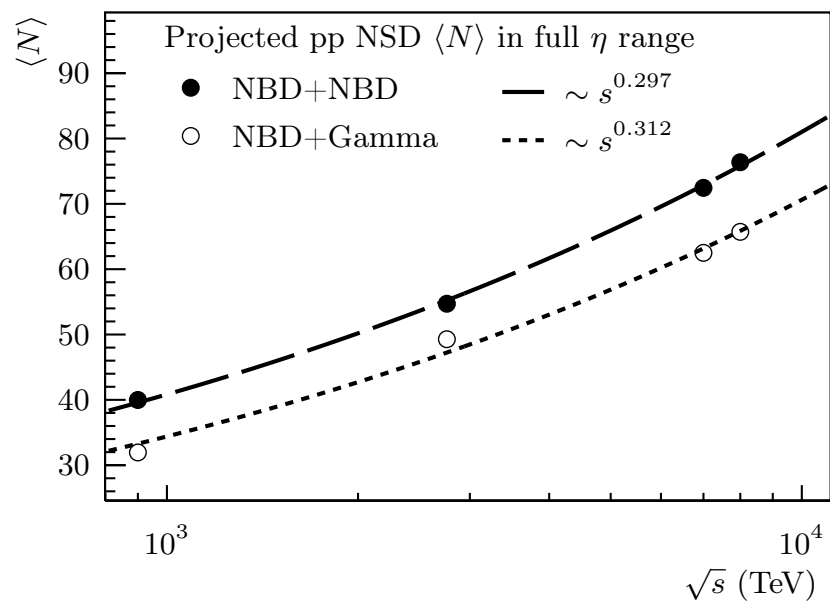

Fig. 4. Estimated unrestricted phase space average multiplicity in proton-proton collisions as a function of the center-ofmass energy $\sqrt{s}$. Powerlaw fits are added to indicate the trend 


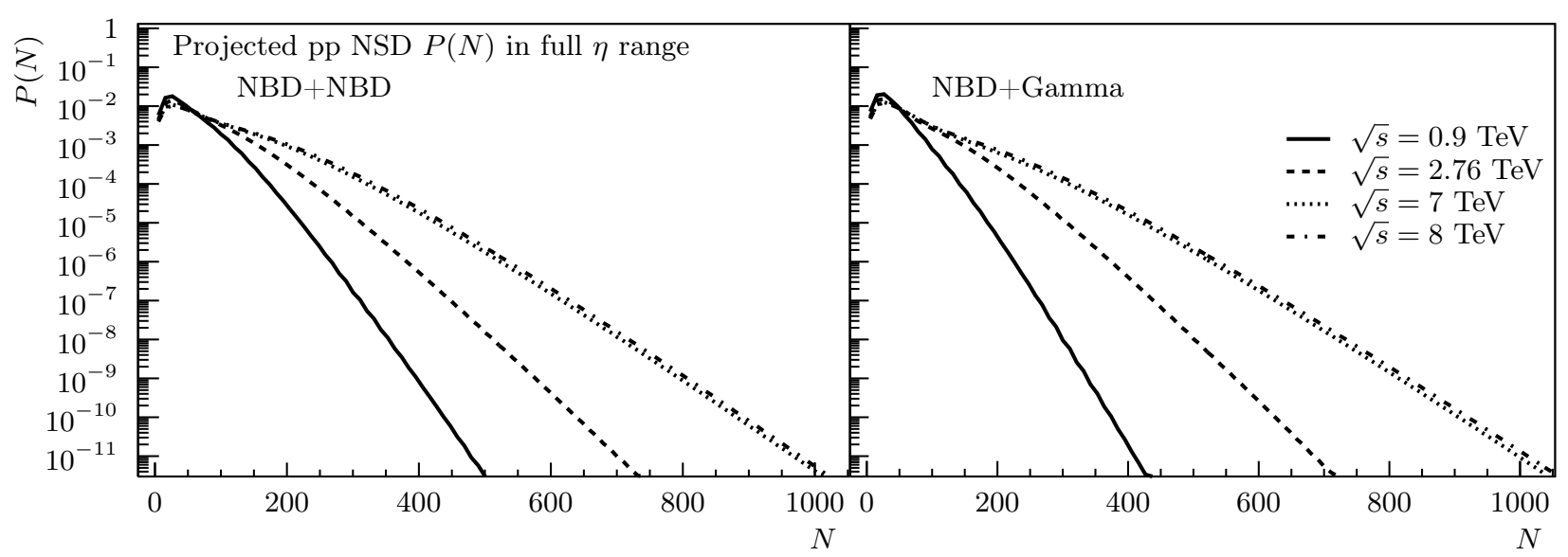

Fig. 5. Unrestricted phase space multiplicity distributions for proton-proton collisions at various energies projected from the $\mathrm{NBD}+\mathrm{NBD}$ (left) and NBD + Gamma (right) models

Table 5. Fit parameters and $\chi^{2}$ for the model fit with the full phase space multiplicity distribution as a weighted sum of two NBDs (presented in Figs. 2 and 3 ) and with a weighted sum of NBD and the gamma distribution

\begin{tabular}{|c|c|c|c|c|c|c|c|c|c|c|}
\hline \multicolumn{11}{|c|}{$\mathrm{NBD}+\mathrm{NBD}$} \\
\hline$\sqrt{s}(\mathrm{TeV})$ & $\alpha$ & $\langle N\rangle_{1}$ & $k_{1}$ & $\langle N\rangle_{2}$ & $k_{2}$ & $A_{1}$ & $A_{2}$ & $A_{3}$ & $\chi^{2} / \mathrm{NDF}$ & Ratio $A_{1}: A_{2}: A_{3}$ \\
\hline 0.9 & 0.55 & 23.92 & 3.23 & 59.29 & 3.79 & 0.103 & 0.204 & 0.301 & $81.517 / 160$ & $1.000: 1.986: 2.941$ \\
\hline 2.76 & 0.59 & 31.16 & 2.55 & 87.80 & 3.69 & 0.092 & 0.182 & 0.268 & $116.085 / 230$ & $1.000: 1.979: 2.907$ \\
\hline 7 & 0.66 & 41.49 & 1.91 & 132.63 & 4.06 & 0.084 & 0.167 & 0.249 & $212.602 / 328$ & $1.000: 1.990: 2.965$ \\
\hline 8 & 0.68 & 45.35 & 1.89 & 143.20 & 4.47 & 0.085 & 0.168 & 0.247 & $259.175 / 314$ & $1.000: 1.980: 2.910$ \\
\hline \multicolumn{11}{|c|}{$\mathrm{NBD}+$ Gamma } \\
\hline$\sqrt{s}(\mathrm{TeV})$ & $\alpha$ & $\langle N\rangle_{1}$ & $k_{1}$ & $k_{2}$ & $\langle N\rangle_{2}$ & $A_{1}$ & $A_{2}$ & $A_{3}$ & $\chi^{2} / \mathrm{NDF}$ & Ratio $A_{1}: A_{2}: A_{3}$ \\
\hline 0.9 & 0.80 & 25.98 & 2.28 & 4.00 & 55.32 & 0.128 & 0.252 & 0.376 & $196.316 / 160$ & $1.000: 1.979: 2.945$ \\
\hline 2.76 & 0.70 & 30.39 & 2.50 & 4.00 & 93.34 & 0.096 & 0.189 & 0.279 & $76.353 / 230$ & $1.000: 1.972: 2.916$ \\
\hline 7 & 0.80 & 42.68 & 1.93 & 4.00 & 140.15 & 0.087 & 0.171 & 0.257 & $792.772 / 328$ & $1.000: 1.962: 2.960$ \\
\hline 8 & 0.76 & 41.84 & 1.97 & 4.00 & 141.34 & 0.088 & 0.172 & 0.256 & $270.035 / 314$ & $1.000: 1.963: 2.920$ \\
\hline
\end{tabular}

tion suggests. Alternatively, it can indicate the inadequacy of the shape universality assumption, which can be tested, by using data from wider pseudorapidity intervals.

Finally, the possibility of the internal inconsistency between ALICE multiplicity measurements at different energies cannot be discarded. In particular, the direct fits, both from ALICE and performed here, produce notably different parameters (specifically, the weight $\alpha$ and shape parameters $k_{1,2}$ ) between measurements at 7 and $8 \mathrm{TeV}$. As there is no expectation for measurements being considerably different with just the 14-energy (and completely negligible on the $\ln s$ scale of high-energy physics), such properties can be both explained with the internal inconsistency and the appearance of new scattering modes at these energies. Note that the stability of the gamma component $k$ parameter observed for direct fits still holds for the combined model, though with a less compatibility to data. This may be changed, when the anal- 
ysis is performed with more pseudorapidity intervals, when they become available. Regardless, such stability is an indication of that a subsample of events governed by a particular particle production mechanism exhibits the scaling behavior already at $\mathrm{TeV}$ energies. A more detailed analysis is required. However, we will again point out that the most significant problem of multiplicity measurements is the correlated uncertainty. The lack of a direct information on correlations removes a number of statistical tools at our disposal, which can be used to extract physical results in a straightforward way.

Note that we are not modeling the subsample of events that gives rise to the "S" shape at low multiplicities becoming more pronounced with increasing the $\eta$ range, which obviously has a different shape of the pseudorapidity density. The inclusion of additional terms in $P_{\text {tot }}(N)$ at this stage would not improve the analysis. However, it may be required to describe wider $\eta$ intervals.

A possible expansion of the method suggested here would include the simultaneous modeling of both pseudorapidity density and unrestricted phase space multiplicity distribution, ultimately without the factorization assumption used to construct $P(n \mid N, \Delta \eta)$.

\section{Conclusion}

The phenomenological model presented in this paper is able to successfully simultaneously describe several multiplicity distributions in varying pseudorapidity intervals at a given energy. Using experimental distributions in more pseudorapidity intervals, this can be improved further by constraining the weight parameter $\alpha$ that controls the sample separation, and the shapes of separate distributions of the either sample controlled by the parameters $k_{1,2}$. Such approach paves a way to making charged-particle multiplicity distributions more useful as a tool to probe, in particular, the balance between soft- and hard-QCD processes in collider experiments, as it leaves less free parameters to describe phenomenologically or theoretically. As demonstrated by the analysis performed, such unified approach already allows a better sample separation than the direct fitting of the shapes of restricted phase space multiplicity distributions, though it is still limited by unknown correlations in the experimental systematic uncertainty. An important consequence of this is the potential for developing the experimental techniques to measure overall multiplicity distributions, as well as an additional sample separation criterion for the protonproton scattering that lacks a clear analog of the ionion physics centrality.

It is shown that charged particle multiplicity distributions at energies ranging from 0.9 to $8 \mathrm{TeV}$ are well described by the model that assumes the uncorrelated mix of two event samples, with different multiplicity distribution shapes, both given by the negative-binomial distribution. The gamma distribution, which can be regarded as the scaling limit of NBD, is also compatible with the second sample, at least at higher energies, by suggesting that the scaling behavior may manifest itself in different event samples at different $\sqrt{s}$, by explaining the apparent lack of the overall scaling. It can be suggested that, as the center-of-mass energy and, thus, the average multiplicity increase, by leading to $\langle n\rangle / k \gg 1$, both components will become compatible with the gamma distribution reaching the corresponding scaling limits.

The shape of the particle pseudorapidity density distribution plays an important role within this framework. If the experimental results for the pseudorapidity density in proton-proton collisions, classified somehow in bins by the overall event multiplicity (or related inelasticity measure) become available, it will be possible to refine this model, by possibly including the dependence of the restriction factors $A$ on the total number of particles $N$. However, even the usual averaged pseudorapidity distribution measured in a wider $\eta$ interval can be used to extract more precise values of $A$, by constraining the rest of model parameters.

One of the important consequences of the proposed model is the stability of the NBD shape parameter $k$ across different pseudorapidity intervals, which contradicts a long-standing assumption based on the fact that direct fits yield different values for different $\eta$ ranges. Moreover, this parameter, through its relation to the dispersion of NBD, is also directly related to the two-particle correlation function [11]. Thus, this observation potentially has significant theoretical consequences.

Overall, the described approach offers a novel view of restricted phase space charged particle multiplicity distributions that has a lot of potential. This model will be applied to upcoming experimental data, and we will continue our work on refining different aspects of it, in particular, by combining more sources of in- 
formation about the particle production in protonproton collisions.

The author wishes to express his gratitude to G.M.Zinovjev, Ye.S.Martynov, and Jean-Pierre Revol for their support and fruitful discussions.

This study is performed in a framework of the targeted research program of the Division of Physics and Astronomy of the National Academy of Sciences of Ukraine.

1. M.L. Miller, K. Reygers, S.J. Sanders, P. Steinberg. Glauber modeling in high energy nuclear collisions. Ann. Rev. Nucl. Part. Sci. 57, 205 (2007), arXiv:nucl-ex/0701025 [nucl-ex].

2. J. Adam et al. (ALICE). Enhanced production of multistrange hadrons in high-multiplicity proton-proton collisions. Nature Phys. 13, 539 (2017), arXiv:1606.07424 [nuclex].

3. A. Alkin, E. Martynov, O. Kovalenko, S.M. Troshin. Impact-parameter analysis of TOTEM data at the LHC: Black disk limit exceeded. Phys. Rev. D 89, 091501 (2014), arXiv:1403.8036 [hep-ph].

4. P. Carruthers, C.C. Shih. The phenomenological analysis of hadronic multiplicity distributions. Int. J. Mod. Phys. A 2, 1447 (1987).

5. C. Fuglesang. UA5 multiplicity distributions and fits of various functions. In Multiparticle dynamics: A meeting ground between particle and statistical physics. A dialog between experiment and theory. Festschrift for Leon van Hove. Proceedings of the Conference at La Thuile, Italy (1989).

6. K. Aamodt et al. (ALICE). Charged-particle multiplicity measurement in proton-proton collisions at $\sqrt{s}=0.9$ and $2.36 \mathrm{TeV}$ with ALICE at LHC. Eur. Phys. J. C 68, 89 (2010), arXiv:1004.3034 [hep-ex].

7. V. Khachatryan et al. (CMS). Charged particle multiplicities in pp interactions at $\sqrt{s}=0.9,2.36$, and $7 \mathrm{TeV}$. JHEP 1101, 079 (2011), arXiv:1011.5531 [hep-ex].

8. J. Adam et al. (ALICE). Charged-particle multiplicities in proton-proton collisions at $\sqrt{s}=0.9$ to $8 \mathrm{TeV}$. Eur. Phys. J. C 77, 33 (2017), arXiv:1509.07541 [nucl-ex].

9. V. Zaccolo (ALICE). Charged-particle multiplicity distributions over a wide pseudorapidity range in proton-proton collisions with ALICE. Nucl. Phys. A 956, 529 (2016), arXiv:1512.05273 [hep-ex].

10. B. Abelev et al. (ALICE). Measurement of inelastic, singleand double-diffraction cross sections in proton-proton collisions at the LHC with ALICE. Eur. Phys. J. C 73, 2456 (2013), arXiv:1208.4968 [hep-ex].

11. A. Giovannini, L. Van Hove. Negative binomial multiplicity distributions in high-energy hadron collisions. Z. Phys. C 30, 391 (1986).

12. A. Polyakov. A similarity hypothesis in the strong interactions. 1. Multiple hadron production in $\mathrm{e}^{+} \mathrm{e}^{-}$annihilation. Sov. Phys. JETP 32, 296 (1971).
13. Z. Koba, H.B. Nielsen, P. Olesen. Scaling of multiplicity distributions in high-energy hadron collisions. Nucl. Phys. $B$ 40, 317 (1972).

14. A. Breakstone et al. (Ames-Bologna-CERN-DortmundHeidelberg-Warsaw). Charged multiplicity distribution in pp interactions at ISR energies. Phys. Rev. D 30, 528 (1984).

15. A. Giovannini, R. Ugoccioni. Possible scenarios for soft and semihard components structure in central hadron hadron collisions in the $\mathrm{TeV}$ region. Phys. Rev. D 59, 094020 (1999), arXiv:hep-ph/9810446.

16. A. Giovannini, R. Ugoccioni. Possible scenarios for soft and semihard components structure in central hadronhadron collisions in the $\mathrm{TeV}$ region: Pseudorapidity intervals. Phys. Rev. D 60, 074027 (1999).

17. P. Ghosh. Negative binomial multiplicity distribution in proton-proton collisions in limited pseudorapidity intervals at LHC up to $\sqrt{s}=7 \mathrm{TeV}$ and the clan model. Phys. Rev. $D$ 85, 054017 (2012), arXiv:1202.4221 [hep-ph].

18. G. Wilk, Z. Włodarczyk. How to retrieve additional information from the multiplicity distributions. J. Phys. G 44, 015002 (2017), arXiv:1601.03883 [hep-ph].

19. G. Wilk, Z. Włodarczyk. Oscillations in counting statistics. In Proceedings, 46th International Symposium on Multiparticle Dynamics (ISMD 2016): Jeju Island, South Korea (2017), vol. 141, p. 01005, arXiv:1610.01890 [hep-ph].

20. G. Cowan. Statistical Data Analysis (Clarendon Press, 1998) [ISBN: 9780198501565].

21. A. Alkin, E. Martynov, V. Pauk, E. Romanets. Inclusive distributions in the unitarized pomeron models (2009), arXiv:0911.4698 [hep-ph].

22. V. Pauk, A. Alkin, E. Martynov, E. Romanets. Oneparticle inclusive distribution in the unitarized pomeron models. In Proceedings, 20th Symposium on Hadron collider physics (HCP 2009): Evian, France, November 1620 (2009), vol. HCP2009, p. 091.

Received 26.06.17

A. Алькін

ФЕНОМЕНОЛОГІЯ РОЗПОДІЛІВ

МНОЖИННОСТІ ЗАРЯДЖЕНИХ ЧАСТИНОК

$\mathrm{P}$ е $з$ ю м е

Розподіли множинності заряджених частинок є цікавим інструментом для вивчення як м'яких, так й жорстких КХДпроцесів у адронному розсіянні. 3 попереднього сторіччя значний діапазон енергій зіткнення було експериментально розглянуто, від декількох ГеВ до $13 \mathrm{TeB}$ в останньому періоді роботи LHC. Сукупний аналіз розподілів множинності в зіткненнях протонів на різних енергіях, в різних регіонах фазового простору народжених частинок та від різних експериментальних колаборацій дає можливість систематизувати та перевірити наявні феноменологічні моделі множинного народження частинок. В цій роботі запропоновано феноменологічну модель, що дозволяє одночасно описати розподіли множинності заряджених частинок у зіткненнях протонів у різних інтервалах псевдохуткості. Модель успішно застосовано до експериментальних розподілів множинності, що отримані колаборацією ALICE на LHC.

ISSN 2071-0194. Ukr. J. Phys. 2017. Vol. 62, No. 9 